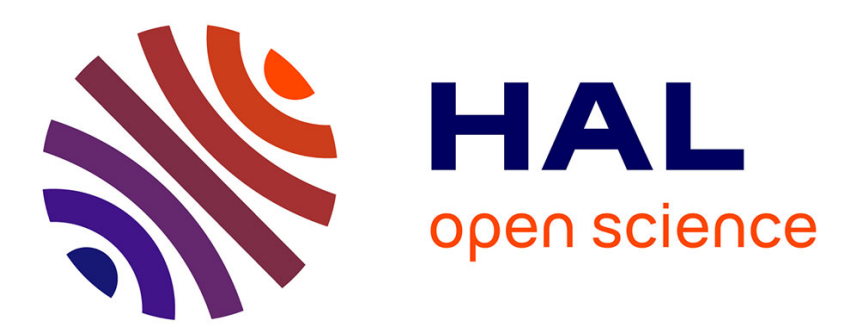

\title{
Surface perturbation inverted from angle variations of eigenbeams in an ultrasonic waveguide
}

\author{
Tobias van Baarsel, Philippe Roux, Jerome I. Mars, Barbara Nicolas
}

\section{To cite this version:}

Tobias van Baarsel, Philippe Roux, Jerome I. Mars, Barbara Nicolas. Surface perturbation inverted from angle variations of eigenbeams in an ultrasonic waveguide. Journal of the Acoustical Society of America, 2020, 148, pp.2841 - 2850. 10.1121/10.0002447 . hal-03052309

\section{HAL Id: hal-03052309 \\ https://hal.science/hal-03052309}

Submitted on 10 Dec 2020

HAL is a multi-disciplinary open access archive for the deposit and dissemination of scientific research documents, whether they are published or not. The documents may come from teaching and research institutions in France or abroad, or from public or private research centers.
L'archive ouverte pluridisciplinaire HAL, est destinée au dépôt et à la diffusion de documents scientifiques de niveau recherche, publiés ou non, émanant des établissements d'enseignement et de recherche français ou étrangers, des laboratoires publics ou privés. 


\section{Surface perturbation inverted from angle variations of eigenbeams in an ultrasonic waveguide}

Tobias van Baarsel, Philippe Roux, Jérôme Igor Mars, and Barbara Nicolas

Citation: The Journal of the Acoustical Society of America 148, 2841 (2020); doi: 10.1121/10.0002447

View online: https://doi.org/10.1121/10.0002447

View Table of Contents: https://asa.scitation.org/toc/jas/148/5

Published by the Acoustical Society of America

\section{ARTICLES YOU MAY BE INTERESTED IN}

Characterization of seafloor roughness from high-frequency acoustic backscattering measurements in shallow water off the west coast of India

The Journal of the Acoustical Society of America 148, 2987 (2020); https://doi.org/10.1121/10.0002354

How loud is the underwater noise from operating offshore wind turbines?

The Journal of the Acoustical Society of America 148, 2885 (2020); https://doi.org/10.1121/10.0002453

Time-domain room acoustic simulations with extended-reacting porous absorbers using the discontinuous Galerkin method

The Journal of the Acoustical Society of America 148, 2851 (2020); https://doi.org/10.1121/10.0002448

Woodwind instrument design optimization based on impedance characteristics with geometric constraints The Journal of the Acoustical Society of America 148, 2864 (2020); https://doi.org/10.1121/10.0002449

Directional sound source modeling using the adjoint Euler equations in a finite-difference time-domain approach The Journal of the Acoustical Society of America 148, 3075 (2020); https://doi.org/10.1121/10.0002425

Evaluating temporary threshold shift onset levels for impulsive noise in seals

The Journal of the Acoustical Society of America 148, 2973 (2020); https://doi.org/10.1121/10.0002649

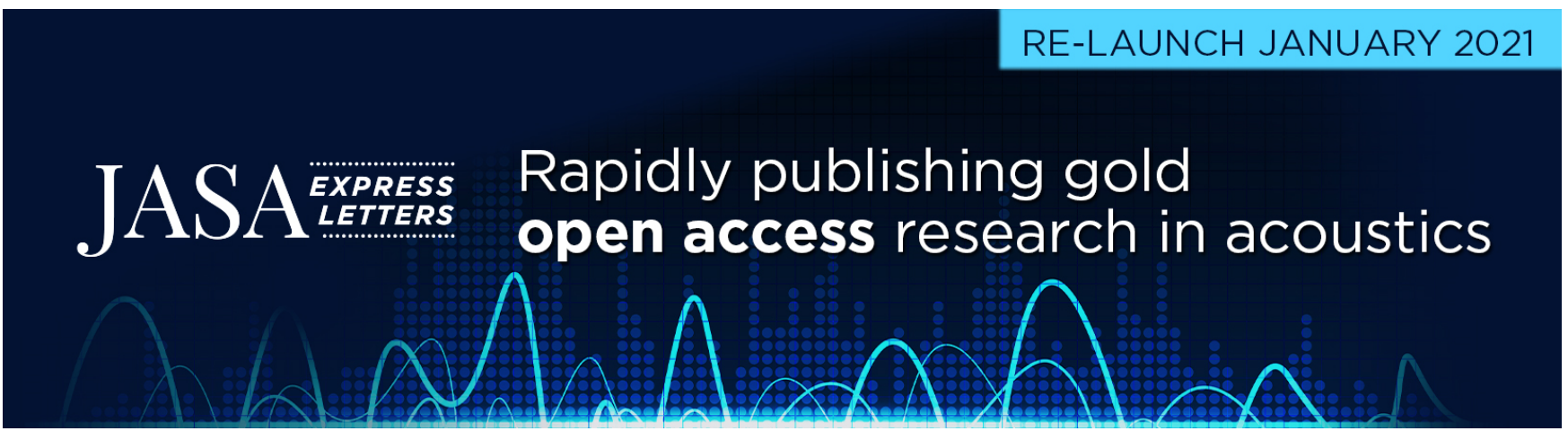




\title{
Surface perturbation inverted from angle variations of eigenbeams in an ultrasonic waveguide
}

\author{
Tobias van Baarsel, ${ }^{1, a)}$ Philippe Roux, ${ }^{2}$ Jérôme Igor Mars, ${ }^{3}$ and Barbara Nicolas ${ }^{4}$ \\ ${ }^{1}$ INRIA, Université de Bordeaux, campus Talence, 200 Avenue de la Vieille Tour, 33405 Talence, France \\ ${ }^{2}$ Institut des Sciences de la Terre, Université Grenoble Alpes, 1381 rue de la Piscine, Grenoble 38041, France \\ ${ }^{3}$ Univeristy of Grenoble Alpes, CNRS, Grenoble INP, GIPSA-Lab, 38000 Grenoble, France \\ ${ }^{4}$ University of Lyon, INSA-Lyon, Université Claude Bernard Lyon 1, UJM Saint-Etienne, CNRS, Inserm, CREATIS, UMR 5220, U1206, \\ F-69100, Lyon, France
}

\begin{abstract}
:
Ocean acoustic tomography is traditionally performed using the travel-time variations of an acoustic path between a source and a receiver. In the context of shallow-water tomography and multipath propagation, the different acoustic paths can be correctly identified if the source and the receiver are arrays of transducers. Here, a double-beamforming algorithm can be applied to extract a collection of eigenbeams from the raw acoustic dataset. In this study, four observables can be measured for each eigenbeam: the travel-time, the amplitude, and the emitting and receiving angles. In this study, the sensitivity kernel (SK) formulation is used to establish a quantitative relation between a perturbation of the surface of an ultrasonic waveguide and the emitting and receiving angles of each eigenbeam. This theoretical relation is experimentally demonstrated using a forward model experiment designed to measure the SK. The SK formulation is then used in a second experiment to quantitatively and dynamically image the propagation of a surface wave traveling across the surface of the waveguide. The inversion results show that the quality of the joint inversion of the emitting and receiving angles is higher than previous results based on amplitude or travel-time observables. @ 2020 Acoustical Society of America. https://doi.org/10.1121/10.0002447
\end{abstract}

(Received 3 July 2020; revised 6 October 2020; accepted 12 October 2020; published online 24 November 2020)

[Editor: John A. Colosi]

Pages: $2841-2850$

\section{INTRODUCTION}

Oceanic acoustic tomography is traditionally performed based on the travel-time variations of acoustic waves that arise from local fluctuations of the medium velocity (Munk and Wunsch, 1979). These variations are classically modeled using the Fermat principle and are therefore associated with a specific acoustic path (Cornuelle, 1982). In the specific case of a shallow-water acoustic waveguide, the multipath propagation produces a set of acoustic arrivals that can superimpose on the receiver (Roux and Fink, 2000). In the acoustic ray approximation, each of these arrivals corresponds to a different eigenpath between the source and the receiver, with a unique set of emitting angles, receiving angles, and travel-times. In most cases, the emitting and receiving angles are not measurable, as the acoustic source and receiver are generally single transducers.

However, by using arrays of transducers, the angle information linked to the acoustic propagation can be extracted. In the present case, the extraction of the emitting and receiving angles as quantitative observables associated with each individual echo is performed through a doublebeamforming (DBF) algorithm. DBF allows us to project the acoustic pressure field onto the waveguide eigenbeams, thus going from a source-and-receiver sensor space to an

\footnotetext{
${ }^{\text {a)} E l e c t r o n i c ~ m a i l: ~ t o b i a s . v a n-b a a r s e l @ i n r i a . f r ~}$
}

emitting-and-receiving angle space (Roux et al., 2008). In this new space, each eigenbeam is represented by an intensity spot. As the eigenray concept results from the application of ray theory that is traditionally valid in an infinite bandwidth-high frequency approximation, we prefer to describe in the following the ultrasonic arrivals extracted from the DBF process as eigenbeams in the finite-frequency approach.

This projection of the pressure field on the eigenbeam space gives access to a total of four observables that can be used for shallow-water tomography and monitoring: the travel-time, the amplitude of the eigenbeams, and the emitting and receiving angles. Furthermore, it allows the acoustic field to be separated into independent acoustic arrivals that are related to each multipath echo, making the inversion process much easier in the case of a waveguide perturbation.

The inversion of the waveguide perturbation is achieved using sensitivity kernels (SKs) for a given eigenbeam that describe its sensitivity to a given observable. This formulation is based on diffraction physics, and it has been used since the 1980s in geophysics (Snieder and Nolet, 1987), and since the early 2000s in underwater acoustics (Skarsoulis and Cornuelle, 2004). In the context of wave propagation in a shallow-water waveguide, previous studies have dealt with acoustic tomography based on travel-times (Iturbe et al., 2009) or amplitudes (Marandet et al., 2011). 
At the same time, it has been shown that local fluctuations of the propagation medium do not only influence the traveltime, but also the emitting and receiving angles of each eigenbeam (Roux et al., 2008). The variations of the emitting and receiving angles were used jointly with the traveltime by Aulanier et al. (2013) to invert a local perturbation of the sound speed localized in the waveguide.

In this paper, we propose to use only the two new angle observables, the emitting and receiving angles of each eigenbeam, to localize and estimate the perturbation of the air-water interface at the surface of an ultrasonic waveguide. This is achieved using the SKs for the two-dimensional (2D) surface approach, which has been used previously for traveltimes and amplitudes (Roux and Nicolas, 2014; Sarkar et al., 2012; van Baarsel et al., 2019), and is here developed for the eigenbeam angles.

This paper uses two experiments to invert for surface perturbations at the air-water interface of an ultrasonic waveguide using the changes in the incidence angles on the perturbed interface of selected eigenbeams. The first experiment is designed to measure the effects of a known perturbation of the water surface on the emitting and receiving angles of selected eigenbeams inside the waveguide and will therefore be referred to as the forward model (FM) experiment. The goal of the second experiment is to invert an unknown perturbation of the air-water interface using the angle variations of a large set of eigenbeams, and this will be referred to as the inverse problem (IP) experiment. The inversion is performed from a linear algebraic formulation deduced from the SK approach.

\section{EXPERIMENTAL DESIGN}

The two set-ups are two variations of an ultrasonic Pekeris waveguide (Pekeris, 1948). Two source-receiver ultrasonic arrays face each other in a small-scale shallowwater waveguide. The waveguide is $55 \mathrm{~mm}$ deep and $0.6 \mathrm{~m}$ long for the FM experiment, and $55 \mathrm{~mm}$ deep and $1 \mathrm{~m}$ long for the IP experiment. The surface perturbation in the IP experiment is independently measured by optical means for validation purposes (not shown here; see van Baarsel et al., 2019). The optical measurements are carried out using two cameras: one that films the water surface from the top and records the frequency-wavenumber information of the perturbation; the second that views the perturbation from the side and records the height of the perturbation. However, the resolution of this camera is too low for accurate measurements, and this allows only an order of magnitude estimate of the perturbation. Moreover, perturbations of less than $1 \mathrm{~mm}$ cannot be recorded optically, although the comparisons between different experiments with different excitation strengths show the correct estimation on the order of magnitude of the surface displacement, even for very small perturbations (i.e., $\approx 1 e-5 \mathrm{~m}$ ).

The arrays are composed of 64 transducers centered at $1 \mathrm{MHz}$. The transducer dimensions are $0.75 \mathrm{~mm}$ along the vertical axis and $12 \mathrm{~mm}$ along the transverse axis. This feature naturally creates a collimated beam in the waveguide axis direction. The bottom of the waveguide is steel and provides a good reflection of ultrasonic waves (Mayer, 1963).

The FM experiment is detailed in Sarkar et al. (2012). It was designed to measure the surface SK using a $5 \mathrm{~mm}$ lead sphere probe that acts as a point perturbation of the surface [Fig. 1(a)]. The penetration of the sphere into the water is approximately $1 \mathrm{~mm}$, and the horizontal translation of the sphere along the interface is $1 \mathrm{~mm}$. For each position of the sphere, the transfer matrix of the waveguide is recorded, which therefore measures the effects of local displacement of the water surface on the acoustic field in the waveguide, from which the SK can be computed. Note that in the FM experiment, the SK is measured only at each position of the sphere along a one-dimensional (1D) central line at the surface of the waveguide, and not on the whole 2D surface of the waveguide.

The IP experiment is detailed in van Baarsel et al. (2019). The surface of the waveguide is perturbed by a blast wave generated above the surface and at the position $x \approx 0.56 \mathrm{~m}$, by laser-induced breakdown [Fig. 1(b)]. The perturbation is localized and controllable, and results in a circular surface wave. Due to the dimensions of the waveguide, this circular wave is seen by the ultrasonic system as

(a)
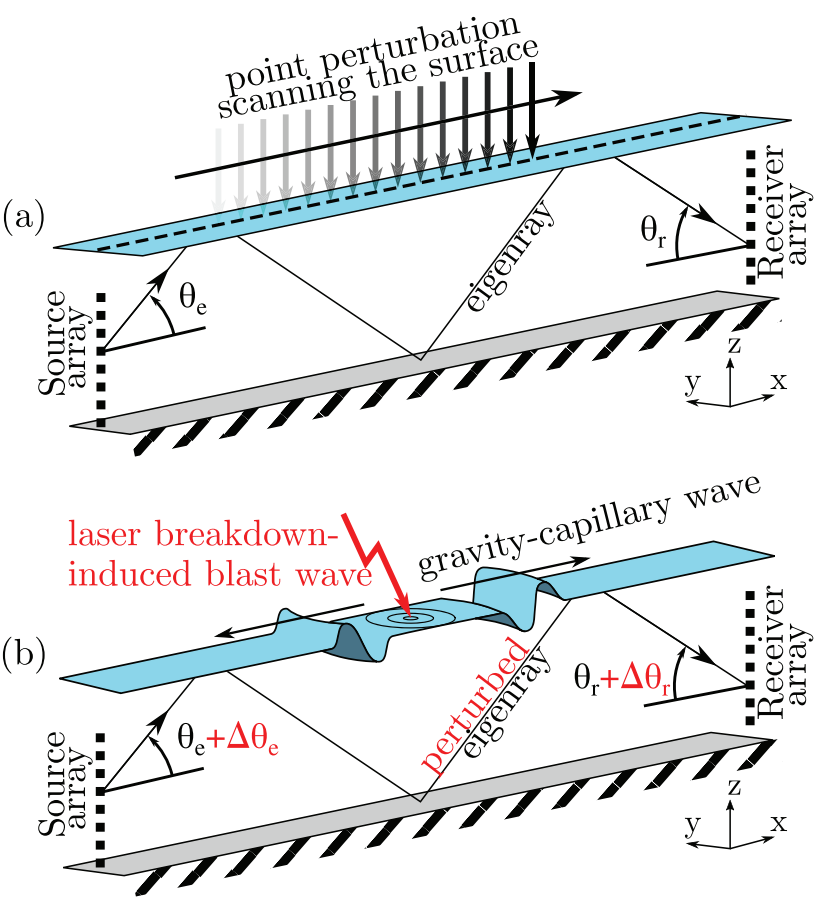

FIG. 1. (Color online) (a) Cartoon of the FM experiment designed to measure the surface SK in a fluid waveguide. A 5-mm lead sphere penetrates the water at different locations on the central line of the surface of the waveguide (gray arrows), and acts as a point perturbation of the water surface. The penetration depth is on the order of $1 \mathrm{~mm}$. The experimental design does not allow the SK to be measured for the entire surface, but only the central line of the surface of the waveguide (dashed line). (b) Cartoon of the IP experiment designed to image a traveling gravity-capillary wave on the surface of the waveguide. The transfer matrix of the waveguide is recorded at a rate of 100 frames/s over $5 \mathrm{~s}$, during which time the surface wave is caused by laser-induced breakdown (lightning-shaped arrow) in the center of the surface of the waveguide. 
two counter-propagating wave packets that expand from the center. While the wave is travelling across the surface, the ultrasonic system records the transfer matrix of the waveguide at 100 times/s, over $5 \mathrm{~s}$.

The transfer matrix of the waveguide is the set of transfer functions for each source-receiver pair ( 64 sources $\times 64$ receivers). As the medium is highly reverberant, a recording window of up to $100 \mu$ s is used, which provides up to 12 distinguishable ultrasonic arrivals for each pair.

The perturbation of the waveguide interface affects each eigenbeam that interacts with the air-water interface. Now we use the SK formalism to describe the variations of each eigenbeam caused by the surface perturbation.

\section{EIGENBEAM ANGLES SKS FOR THE SURFACE PERTURBATION}

This section shows the mathematical relation between a surface perturbation and the variations of the angle observables. The eigenbeams of the waveguide can be separated using the DBF algorithm (Krüger et al., 1993), i.e., a delayand-sum algorithm applied to both the emitting and receiving arrays. The DBF is expressed as

$$
\begin{aligned}
p^{D B F}\left(\theta_{e}, \theta_{r}, t\right)= & \frac{1}{N_{e}} \frac{1}{N_{r}} \sum_{e} \sum_{r} p\left(z_{e}, z_{r}, t\right) \\
& \times \exp \left(-l \omega\left[\tau\left(\theta_{e}, z_{e}\right)-\tau\left(\theta_{r}, z_{r}\right)\right]\right),
\end{aligned}
$$

where $p^{D B F}\left(\theta_{e}, \theta_{r}, t\right)$ is the acoustic pressure in the emitting angle, receiving angle, and time space (DBF space) computed from the acoustic pressure $p\left(z_{e}, z_{r}, t\right.$ ) (in depth and time space) between transducers $z_{e}$ and $z_{r}$ and the number of transducers in the emitting and receiving subarrays $N_{e}$ and $N_{r}$, respectively.

The time delays $\tau\left(\theta_{e}, z_{e}\right)$ and $\tau\left(\theta_{r}, z_{r}\right)$ depend on the local velocity in the propagation medium and the geometry of the emitting and receiving subarrays. In a homogeneous waveguide, these time delays can be written as

$$
\tau(\theta, z)=\frac{\left(z-z_{0}\right) \sin \theta}{c},
$$

where $z-z_{0}$ is the distance between the sensor at depth $z$ and the center of the subarray in $z_{0}, \theta$ is the emitting or receiving angle, and $c$ is the speed of sound.

Note that the homogeneous waveguide approximation is correct in our small-scale water-tank experiment. However, the DBF algorithm can be extended to more complex media by taking into account a depth-dependent soundspeed profile (Aulanier et al., 2013; Roux et al., 2008).

Each eigenbeam corresponds to an ultrasonic arrival and is represented in the DBF space by an intensity spot with a maximum $p^{D B F}\left(\theta_{e}, \theta_{r}, t\right)$ centered on the eigenbeam coordinates $\left[\theta_{e}, \theta_{r}, t\right]$.

For each eigenbeam, the variation of the doublebeamformed pressure $\delta p^{D B F}\left(\theta_{e}, \theta_{e}, t\right)$ is the difference between the pressure $p^{D B F}\left(\theta_{e}, \theta_{r}, t\right)$ in the perturbed waveguide and the rest state $p_{0}^{D B F}\left(\theta_{e}, \theta_{r}, t\right)$ in the unperturbed waveguide. In the small perturbation assumption (van Baarsel et al., 2019), this difference can be linked to the surface perturbation $\Delta h\left(\mathbf{r}^{\prime}\right)$ for each point at the surface $\mathbf{r}^{\prime}$ using the SK formulation, i.e.,

$$
\begin{aligned}
\delta p^{D B F}\left(\theta_{e}, \theta_{r}, t\right)= & p^{D B F}\left(\theta_{e}, \theta_{r}, t\right)-p_{0}^{D B F}\left(\theta_{e}, \theta_{r}, t\right) \\
= & \frac{1}{2 \pi} \iint \Delta h\left(\mathbf{r}^{\prime}\right) K^{D B F}\left(\theta_{e}, \theta_{r}, \mathbf{r}^{\prime}, \omega\right) \\
& \times P_{s}(\omega) \mathrm{e}^{-l \omega t} \mathrm{~d} \omega \mathrm{d} \mathbf{r}^{\prime}
\end{aligned}
$$

where $K^{D B F}$ is the double-beamformed SK for the wavefield amplitude and $P_{S}(\omega)$ is the power spectrum of the source at a given pulsation $\omega$.

The DBF algorithm gives access to four observables for each eigenbeam: the three coordinates of each eigenbeam in DBF space (i.e., the emitting angle $\theta_{e}$, the receiving angle $\theta_{r}$, and the travel-time $t$ ), and the eigenbeam amplitude. Any perturbation of the waveguide surface results in variations of these four observables. Note that the amplitude of the transmitted signal is not a coordinate of the eigenbeam and therefore does not appear directly as such in Eq. (3). The study of the travel-time and amplitude variations $\delta p^{D B F}$ due to a surface perturbation from the SK approach has been discussed previously (Roux and Nicolas, 2014; Sarkar et al., 2012; van Baarsel et al., 2019).

From now on, we limit the present study to the case of a homogeneous waveguide with parallel interfaces, to pursue the analysis with a simplified analytical expression for the double-beamformed SK $K^{D B F}$.

The image theorem allows us to consider each eigenbeam in the unwrapped space with a free space calculation of Green's functions (Fig. 2). In doing so, we do not consider angle-dependent reflection coefficients on the bottom interface, as encountered in a Pekeris waveguide (Pekeris, 1948). Within this approximation that will be discussed later, the expression of the double-beamformed SK can be defined as in Sarkar et al. (2012),

$$
\begin{aligned}
K^{D B F}\left(\theta_{e}, \theta_{r}, \mathbf{r}^{\prime}, \omega\right)= & 4 G_{0}^{B F}\left(\mathbf{r}^{\prime}, \theta_{e}, \omega\right) G_{0}^{B F}\left(\mathbf{r}^{\prime}, \theta_{r}, \omega\right) \\
& \times \frac{\omega^{2}}{c^{2}} \sin \left(\alpha_{e}\right) \sin \left(\alpha_{r}\right),
\end{aligned}
$$

where, as shown in Fig. 2, $\mathbf{r}^{\prime}$ is the position of the surface perturbation for each reflection in the unwrapped space, and $\alpha_{e}$ (respectively, $\alpha_{r}$ ) is the angle between the emitting subarray (respectively, receiving subarray) and $\mathbf{r}^{\prime}$.

In the unwrapped space, $G_{0}^{B F}$ in Eq. (4) can be approximated as the open-field plane-wave Green's function between the emitting subarray (or receiving subarray) and $\mathbf{r}^{\prime}$, of coordinates $\left(r_{x}^{\prime}, r_{z}^{\prime}\right)$, given by

$G_{0}^{B F}\left(\mathbf{r}^{\prime}, \theta_{e}, \omega\right)=(-1)^{n} \exp \left(\imath k\left[r_{x}^{\prime} \cos \theta_{e}+\left(z_{e}-r_{z}^{\prime}\right) \sin \theta_{e}\right]\right)$,

$$
\begin{aligned}
& G_{0}^{B F}\left(\mathbf{r}^{\prime}, \theta_{r}, \omega\right) \\
& \quad=(-1)^{n} \exp \left(\imath k\left[\left(\ell-r_{x}^{\prime}\right) \cos \theta_{r}+\left(z_{r, \text { image }}-r_{z}^{\prime}\right) \sin \theta_{r}\right]\right),
\end{aligned}
$$




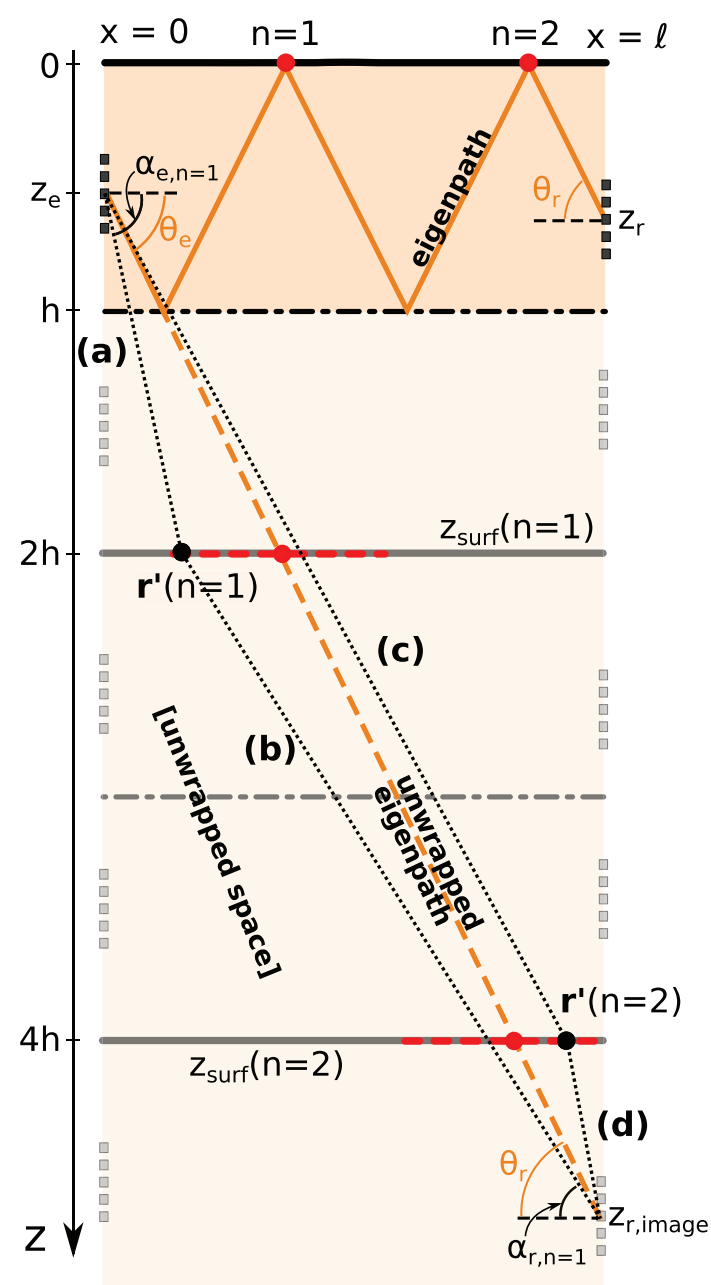

FIG. 2. (Color online) Cartoon of the unwrapping of the waveguide for an eigenray path including $n=2$ reflections at the waveguide surface, represented by an $(x-z)$ slice. The waveguide surface is represented by the horizontal solid line, and the waveguide bottom is the horizontal dashed-dotted line. The unwrapped space is shown in a lighter color than the real waveguide space. The real eigenpath is represented by the solid orange line, whereas the dashed orange line represents the unwrapped eigenpath. The Green's functions (dotted lines) between the emitting array and $\mathbf{r}^{\prime}$ on the one side, and the receiving array and $\mathbf{r}^{\prime}$ on the other side are computed separately for each reflection ( $n=1$ or $n=2)$ on the waveguide interface. (a) $G_{0}^{B F}\left(\theta_{e}, \mathbf{r}^{\prime}, \omega ; n=1\right)$. (b) $G_{0}^{B F}\left(\theta_{r}, \mathbf{r}^{\prime}, \omega ; n=1\right)$. (c) $G_{0}^{B F}\left(\theta_{e}, \mathbf{r}^{\prime}, \omega ; n=2\right)$. (d) $G_{0}^{B F}\left(\theta_{r}, \mathbf{r}^{\prime}, \omega ; n=2\right)$. The angles $\alpha_{e}$ and $\alpha_{r}$ are shown for the first reflection $(n=1)$ on the waveguide interface. The red dots highlight the reflection points at the surface for the unwrapped eigenpath. The red horizontal dashed lines represent the region for which the SK associated with each reflection is computed.

where $\ell$ is the length of the waveguide, $k$ is the acoustic wavenumber, and $n$ is the number of crossings at the airwater interface that takes into account the sign change of the reflected wave in the expression of the unwrapped Green's function $G_{0}^{B F}$. The $z$-coordinate of the receiving subarray center $z_{r \text {,image }}$ and the coordinate $r_{z}^{\prime}$ of each reflection point along the interface are defined in Appendix A.

In practice, the eigenbeam SK $K^{D B F}$ is the summation of the SKs computed for each different surface reflection in the unwrapped space. This is shown in Fig. 2, where the Green's functions $G_{0}^{B F}$ in Eq. (4) are computed separately for $n=1$ (with $\mathbf{r}^{\prime}$ varying along the interface $z_{\text {surf }}=2 h$ ) and $n=2$ (with $\mathbf{r}^{\prime}$ varying along the interface $z_{\text {surf }}=4 h$ ). Note that due to the bandwidth of the acoustic signal, the oscillations of the SK do not extend over the whole interface between $x=0$ and $x=l$ (van Baarsel et al., 2019). Therefore, we can limit each SK computation to around the corresponding reflection point in the unwrapped space [red dashed lines at $z=z_{\text {surf }}(n=1)$ and $z=z_{\text {surf }}(n=2)$ in Fig. 2].

From this point on, the goal of this section is to establish the relation between a surface perturbation $\Delta h\left(\mathbf{r}^{\prime}\right)$ and the variation of the emitting and receiving angles of each eigenbeam in the framework of the SK approach.

The solution follows the methodology defined in Skarsoulis and Cornuelle (2004) and Aulanier et al. (2013). In the $\left[\theta_{e}, \theta_{r}, t\right]$ space, an eigenbeam is a local maximum of the DBF pressure field, i.e.,

$$
\nabla p_{0}\left(\theta_{e}, \theta_{r}, t\right)=\left[\begin{array}{c}
\partial / \partial \theta_{e} \\
\partial / \partial \theta_{r} \\
\partial / \partial t
\end{array}\right] p_{0}\left(\theta_{e}, \theta_{r}, t\right)=\overrightarrow{0}
$$

where $\nabla$ is the gradient operator.

When the surface of the waveguide undergoes a local displacement $\Delta h\left(\mathbf{r}^{\prime}\right)$, the coordinates of the eigenbeam are shifted by the small amounts $\Delta \theta_{e}, \Delta \theta_{r}$, and $\Delta t$, and thus,

$$
\nabla p\left(\theta_{e}+\Delta \theta_{e}, \theta_{r}+\Delta \theta_{r}, t+\Delta t\right)=\overrightarrow{0} .
$$

A first-order Taylor development of Eq. (7) leads to

$$
\begin{aligned}
& \nabla p\left(\theta_{e}, \theta_{r}, t\right)+\Delta \theta_{e} \frac{\partial}{\partial \theta_{e}} \nabla p\left(\theta_{e}, \theta_{r}, t\right) \\
& +\Delta \theta_{r} \frac{\partial}{\theta_{r}} \nabla p\left(\theta_{e}, \theta_{r}, t\right) \\
& +\Delta t \frac{\partial}{\partial t} \nabla p\left(\theta_{e}, \theta_{r}, t\right)=\overrightarrow{0} .
\end{aligned}
$$

Eq. (8) can then be rewritten using the matrix formulation,

$$
\nabla p+\mathbf{H}(p)\left[\Delta \theta_{e}, \Delta \theta_{r}, \Delta t\right]^{T}=\overrightarrow{0},
$$

where $\mathbf{H}(p)$ is the Hessian matrix of $p$, and $[\cdot]^{T}$ is the transpose operator. The expression of the Hessian matrix $\mathbf{H}(p)$ is given in Appendix B. In practice, the Hessian matrix corresponds to the curvature of the DBF pressure field at its maximum; i.e., at the eigenbeam coordinates. As $\mathbf{H}(p)$ $=\mathbf{H}\left(p_{0}\right)+\mathbf{H}(\delta p)$ with $\mathbf{H}(\delta p)$ that is first order in $\Delta h$ [see Eq. (3)]. We can further simplify Eq. (9) by applying the Hessian only to the unperturbed wavefield $p_{0}$. For a small surface perturbation, $\mathbf{H}(p) \approx \mathbf{H}\left(p_{0}\right)$ means that as a first approximation, the intensity maximum described as the eigenbeam is shifted in time and angles but keeps the same shape and therefore the same curvature in the $\left[\theta_{e}, \theta_{r}, t\right]$ space [for experimental validation, see Fig. 4(b) in Roux and Nicolas, 2014].

Since the Hessian matrix is nonzero and invertible for each eigenbeam, we can finally extract the coordinate variations 


$$
\left[\Delta \theta_{e}, \Delta \theta_{r}, \Delta t\right]^{T}=-\mathbf{H}^{-1}\left(p_{0}\right) \nabla(\delta p)
$$

as $\nabla p=\nabla(\delta p)$ from Eq. (6). The calculation of the gradient $\nabla(\delta p)$ is detailed in Appendix C. This leads to an explicit formula for the coordinate variations

$$
\begin{aligned}
{\left[\begin{array}{c}
\Delta \theta_{e} \\
\Delta \theta_{r} \\
\Delta t
\end{array}\right]=} & -\mathbf{H}^{-1}\left(p_{0}\right) \frac{1}{2 \pi} \\
& \times \iint\left[\begin{array}{c}
\imath k\left[-r_{x}^{\prime} \sin \theta_{e}+\left(z_{e}-r_{z}^{\prime}\right) \cos \theta_{e}\right] \\
\imath k\left[-\left(\ell-r_{x}^{\prime}\right) \sin \theta_{r}+\left(z_{r, \text { image }}-r_{z}^{\prime}\right) \cos \theta_{r}\right] \\
-l \omega
\end{array}\right] \\
& \times \Delta h\left(\mathbf{r}^{\prime}\right) K^{D B F}\left(\theta_{e}, \theta_{r}, \mathbf{r}^{\prime}, \omega\right) P_{s}(\omega) \mathrm{e}^{-l \omega t} \mathrm{~d} \omega \mathrm{d} \mathbf{r}^{\prime}
\end{aligned}
$$

As usual, the SK is computed for a broadband signal and at a given time $t$ that corresponds to the travel-time of the specific eigenbeam.

From Eq. (11), we can see that the phase oscillations of the angles kernel are carried by the kernel $K^{D B F}$ and the spatial gradient contribution. On the other hand, the role of the Hessian is to scale the gradient of the SK in all three $\left[\theta_{e}, \theta_{r}, t\right]$ dimensions, by a factor that represents the curvature of the unperturbed eigenbeam in the DBF space.

In Appendix $\mathrm{B}$, the Hessian $\mathbf{H}\left(p_{0}\right)$ and its inverse $\mathbf{H}^{-1}\left(p_{0}\right)$ are obtained in the framework of the plane-wave approximation. In this context, the Hessian is a diagonal matrix that decouples the different observables $\left[\theta_{e}, \theta_{r}, t\right]$. In practice, even if the DBF algorithm helps in the creation of planar wavefronts in the far field, the plane wave approximation should be used with caution in the calculation of the Hessian matrix. What remains from Eq. (B13) in Appendix B, however, is that the angle observables $\left[\theta_{e}, \theta_{r}\right]$ are not coupled with the time observable $t$.

Therefore, limiting our analysis to angle variations only, we can simplify Eq. (11) as

$$
\begin{aligned}
{\left[\begin{array}{c}
\Delta \theta_{e} \\
\Delta \theta_{r}
\end{array}\right]=} & -\frac{1}{p_{0}\left(\theta_{e}, \theta_{r}, t\right)} \frac{3}{N^{3} b^{2}} \frac{1}{2 \pi} \\
& \times \iint\left[\begin{array}{c}
\imath k\left[-r_{x}^{\prime} \sin \theta_{e}+\left(z_{e}-r_{z}^{\prime}\right) \cos \theta_{e}\right] \\
\imath k\left[-\left(\ell-r_{x}^{\prime}\right) \sin \theta_{r}+\left(z_{r, \text { image }}-r_{z}^{\prime}\right) \cos \theta_{r}\right]
\end{array}\right] \\
& \times \Delta h\left(\mathbf{r}^{\prime}\right) K^{D B F}\left(\theta_{e}, \theta_{r}, \mathbf{r}^{\prime}, \omega\right) P_{s}(\omega) \mathrm{e}^{-\imath \omega t} \mathrm{~d} \omega \mathrm{d} \mathbf{r}^{\prime},
\end{aligned}
$$

where $N$ is the number of sensors on the emitting and receiving arrays, and

$$
b=\frac{\pi d}{\lambda} \cos \theta_{e},
$$

where $d$ is the spacing between the elements of the array, and $\theta_{e}$ is the emitting angle of the eigenbeam in the unwrapped space (Fig. 2).

Note that Eq. (12) is equivalent to Eq. (3) for the angle perturbations $\left[\Delta \theta_{e}, \Delta \theta_{r}\right]$ instead of the wavefield amplitude perturbation $\delta p$. As such, it provides an analytical formulation of the SK for angles for a surface perturbation of the waveguide.

Note also that the angle kernels are relative to the amplitude of the unperturbed pressure field $p_{0}\left(\theta_{e}, \theta_{r}, t\right)$ in Eq. (12), which means that they will be no different if our calculation had taken into account reflection coefficients at the waveguide interfaces.

As the purpose of this paper is to invert surface fluctuations from angle variations only, we do not consider from here on the travel-time perturbations in the recovery of the surface perturbation of the waveguide. For discussions about surface fluctuations inverted with travel-time or amplitude perturbations, please refer to previous studies (Roux and Nicolas, 2014; van Baarsel et al., 2019).

A $1 D$ representation of the SK for the emitting and receiving angles is shown in Fig. 3. The predicted SK is compared to the SK measured in the FM experiment. The comparison is satisfactory with respect to the uncertainties in the FM experimental conditions. The uncertainties are mainly the depth of the lead sphere at the air-water interface and the alignment of the experimental SK along the $x$ axis. Therefore, the slight phase and amplitude differences between predicted and experimental SKs in Fig. 3 are balanced out by the large number of SKs used in the inversion process, as will be shown later.

This agreement retrospectively justifies the plane-wave approximation that was carried out (i) in the formulation of the SK in Eqs. (5a) and (5b); (ii) in the calculation of its gradient in Eqs. (C1a) and (C1b); and finally (iii) in the calculation of the Hessian matrix in the Appendix B. This also validates the choice of not taking into account the angledependent reflection coefficient at the waveguide bottom in the computation of the kernel $K^{D B F}$ and its gradient.

A 2D surface representation of the theoretical SKs is shown in Fig. 4. A few points can be noted here. First, the SK for the emitting angle shows higher sensitivity close to the source array. The same behavior is observed for the sensitivity of the receiving angle SK close to the receiver array. Second, we note also the asymmetric shape of the SKs with respect to the reflection point of each eigenbeam at the

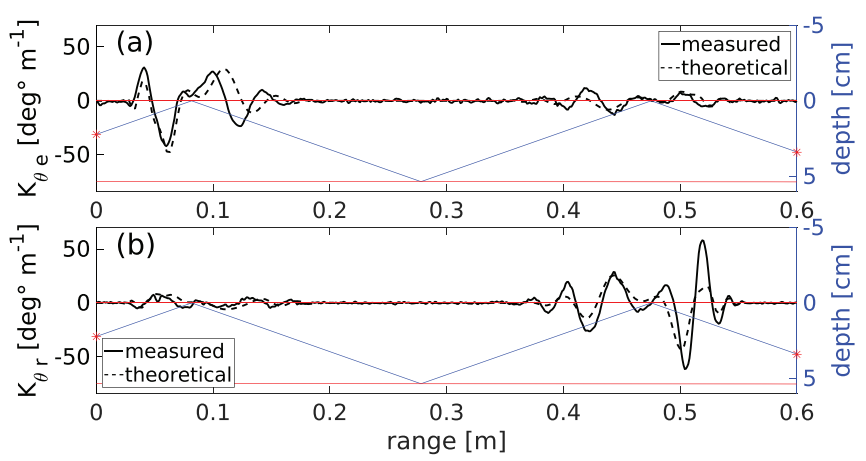

FIG. 3. (Color online) (a) SK for the emitting angle, measured using both the FM experiment (solid line) and computed theoretically (dashed line). Only the central line of the theoretical SK is plotted ( $y=0$; see Fig. 1). The corresponding eigenray $\left(\left[\theta_{e}, \theta_{r}, t\right]=[-14.8 \mathrm{deg},-14.8 \mathrm{deg}, 427.3 \mu \mathrm{s}]\right)$ is depicted under the SK on a different axis. (b) SK for the receiving angle. 

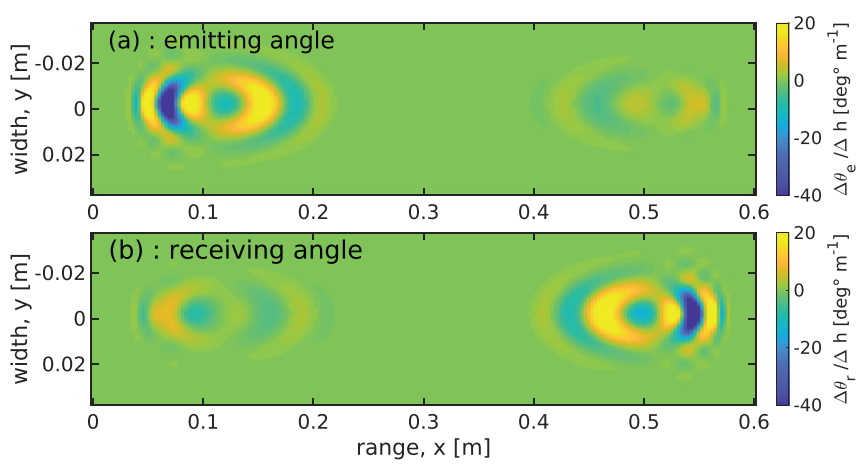

FIG. 4. (Color online) Theoretical SK computed for a local displacement of the surface, for the emitting angle (a), and the receiving angle (b), and for the same eigenray as shown in Fig. 3. Each SK is computed for the entire surface of the waveguide.

interface. Such asymmetry has already been observed for angle SKs predicted for a local sound-speed perturbation inside the waveguide (Aulanier et al., 2013).

In Fig. 7, the amplitude SK established in a previous study (van Baarsel et al., 2019) is plotted for comparison with the angles SK in Fig. 4. Considering the same eigenbeam, the amplitude SK looks particularly different from the emitting-receiving angles SK: (1) the sensitivity of the amplitude is of equal magnitude for the two reflections; and (2) the pattern of the SK amplitude is similar on each side of the reflection point at the interface. As will be discussed later, the shape differences between the angles SK and the amplitude SK results in different spatial resolutions when the inversion process is performed from amplitude or angle observables.

\section{INVERSION RESULTS}

In this section, we present the inversion results from the angle perturbations only, for both the FM and the IP experiments. As in Sarkar et al. (2012) and van Baarsel et al. (2019), a total of 2000 different eigenbeams extracted by the DBF algorithm were combined in the inversion process. The angles SK sets the linear FM between the angle variations $\Delta \theta_{e}$ and $\Delta \theta_{r}$ and the surface displacement $\Delta h$. Using matrix formulation and waveguide discretization, Eq. (12) can be generalized to the full set of eigenbeams as

$$
\Delta \theta=\widehat{K}^{D B F} \Delta h \mathrm{~d} S,
$$

where $\widehat{K}^{D B F}$ refers to the SK for the angles, as derived for a single eigenbeam in Eq. (12).

In the general case, the matrix $\widehat{K}^{D B F}$ does not have an inverse. We use the matrix regularization used in the maximum a posteriori pseudo-inversion scheme described by, e.g., Beydoun and Tarantola (1988) and Roux and Nicolas (2014). The inversion can be achieved using one variable (emitting or receiving angle of the eigenbeams), or the two variables together in a joint inversion.

Therefore, an estimation of the displaced surface $\widehat{\Delta h}$ can be written as

$$
\widehat{\Delta h}=\frac{1}{d S} C_{m} K^{T}\left(K C_{m} K^{T}+C_{d}\right)^{-1} \Delta \theta
$$

where $K=\widehat{K}^{D B F}$ for the sake of simplicity, ${ }^{T}$ is the matrix transpose operator, $C_{m}$ is the covariance matrix of the model, and $C_{d}$ is the covariance matrix of the data. Again, for the sake of simplicity, the data misfits on the beam observables are considered to be independent, i.e., $C_{d}$ is diagonal and $C_{d}=\alpha \mathbb{I}$, where $\alpha$ is the data misfit for all of the eigenbeams, as estimated from the variations in the data when the system is at rest. The model covariance matrix $C_{m}$ is set in such a way that reconstructed surface deformations are spatially correlated with a 2 -cm smoothing distance (Roux and Nicolas, 2014).

\section{A. FM experiment inversion results}

In the FM experiment, the surface of the waveguide is perturbed by a small lead sphere that penetrates the water at different locations successively. For each position of the lead sphere, the inversion of the variations of the ultrasonic field allows the location and magnitude of the surface perturbations to be retrieved. Figure 5 shows the inversion result for the FM experiment, based on the emitting angle [Fig. 5(a)], the receiving angle [Fig. 5(b)], and both variables together using joint inversion [Fig. 5(c)].

The inversion result is a matrix with the highest values on the main diagonal, which shows that the position of the perturbation is correctly estimated. Furthermore, the estimation of the height of the surface perturbation $\widehat{\Delta h}$ matches the penetration depth of the lead sphere.

The joint inversion [Fig. 5(c)] combines both the emitting and receiving angles, and shows better results than the single-variable inversions: the estimation of the location of the perturbation is accurate for the whole range of the waveguide and shows less faulty surface displacements in the upper and lower triangles of the inversion result matrix. Thus, the FM experiment confirms that the use of joint inversion of two independent angle observables provides significant improvement over the inversion of a single angle observable.

Finally, the inversion result of the FM experiment validates the use of the SK formalism for the emitting and receiving eigenbeam angles in the case of a small, singlepoint perturbation. It has been shown previously (van Baarsel et al., 2019) that the SK formalism applied to eigenbeam amplitude variations succeeds in inverting the dynamics of two counter-propagating gravity-capillary wave packets excited by laser-induced breakdown. Taking this previous work a step further, the new SK formalism is now applied to the eigenbeam angle variations induced by the same laboratory-scale experiment.

\section{B. IP experiment; inversion results}

The IP experiment uses laser-induced breakdown to generate a point perturbation of the water surface. The point perturbation evolves into gravity-capillary waves that 
(a) : emitting angle

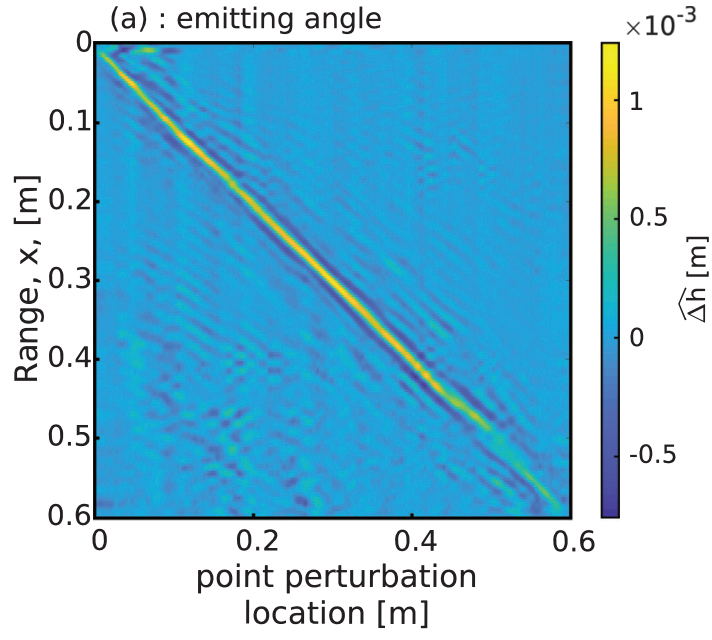

(b) : receiving angle

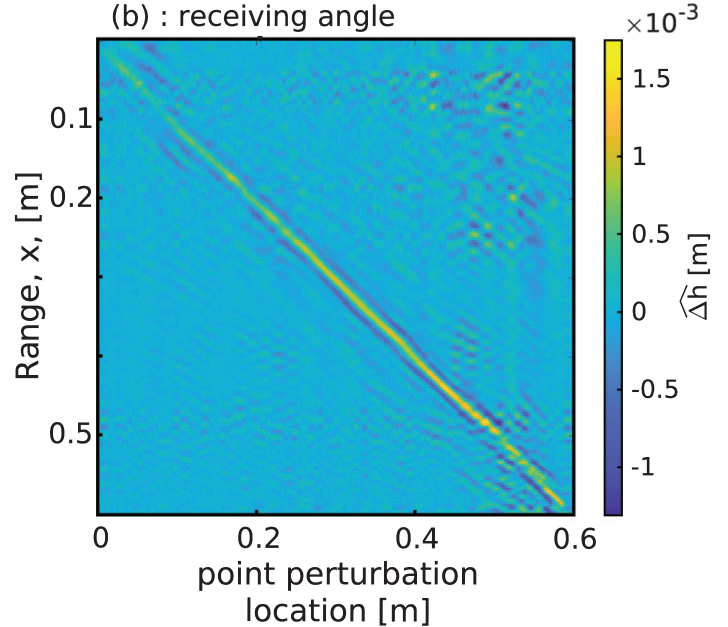

(c) joint inversion

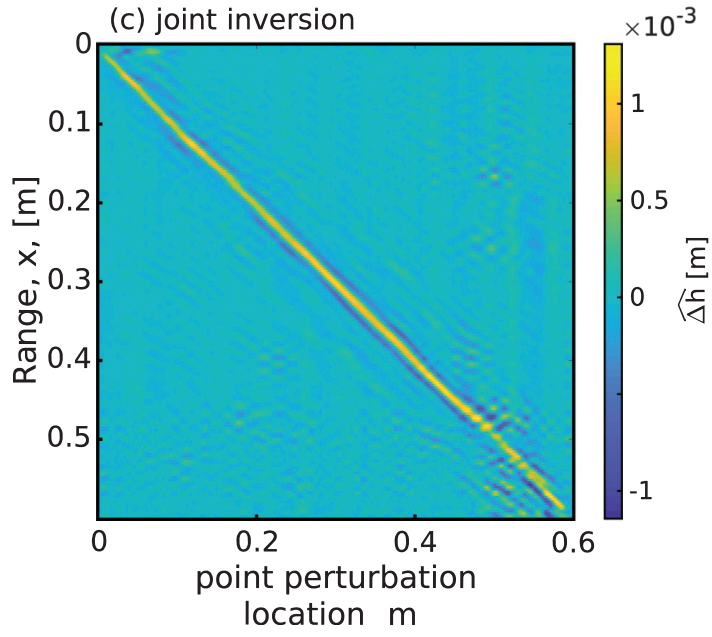

FIG. 5. (Color online) Water surface displacement $\widehat{\Delta h}$ for a point perturbation scanning the surface, inverted using the corrected SK for the emitting angle (a), the receiving angle (b), and both the emitting and receiving angles (c) of a collection of 1292 eigenbeams. All three inversions show good agreement with the experiment, by estimating a point perturbation of $\widehat{\Delta h} \approx 1 \mathrm{~mm}$.

propagate in all directions across the surface. Due to the geometry of the waveguide, these circular waves are seen by the acoustic imaging system as two counter-propagating wave packets. The result of the joint angles inversion of the water height displacement is shown in Fig. 6(a). a) : joint angle inversion

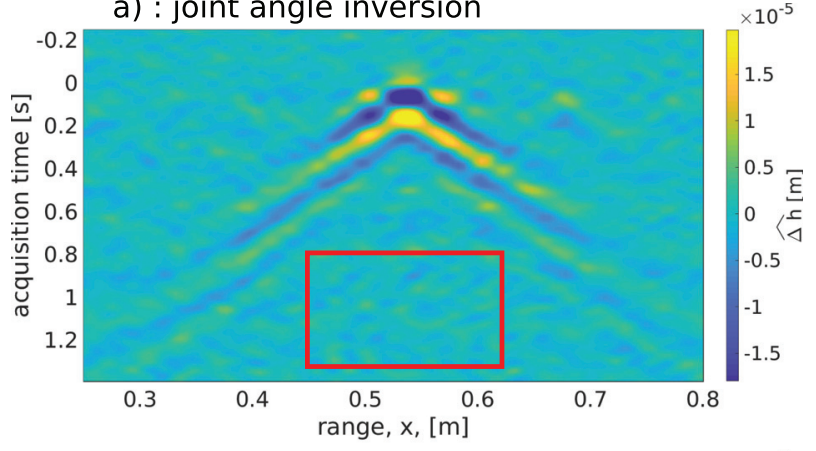

b) : amplitude inversion

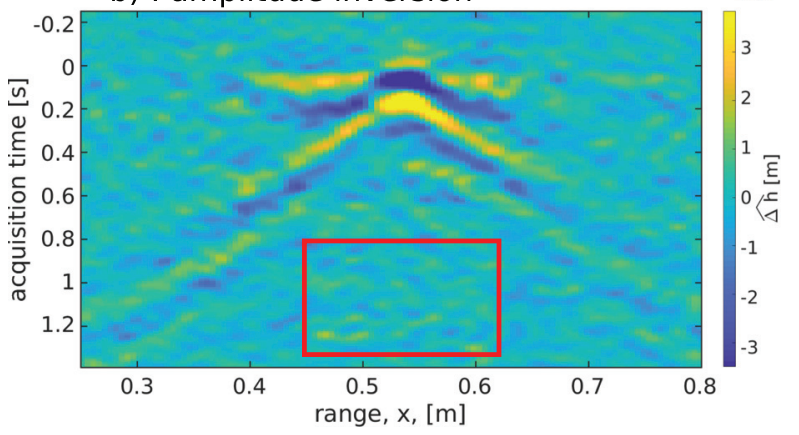

FIG. 6. (Color online) (a) Inversion result of the traveling surface-wave experiment using both emitting and receiving angles of a collection of 2285 eigenbeams. The $x$ axis is the length of the waveguide; the $y$ axis is the time relative to the laser shot that causes the surface wave. (b) Figure reproduced from van Baarsel et al. (2019). Inversion result of the traveling surfacewave experiment using the amplitude variation $\triangle A / A$ of the same collection of eigenbeams. The red rectangles in (a) and (b) represent the area used for the calculation of the inversion residue variance.

The inversion is performed on the whole surface of the waveguide, but for the sake of the representation here, the inversion result is plotted as a function of the acquisition time and the waveguide range. Therefore, only the displacement at the central line of the surface $(y=0)$ is shown.

In Fig. 6(a), the point-like perturbation at $t=0$ can be clearly seen, as for the two wave packets that propagate in opposite directions. The dynamic inversion of the waveguide interface from the angle variations is to be compared with the inversion result of the same experiment performed from the eigenbeam amplitude variation (the "low power" experiment described in van Baarsel et al., 2019) shown in Fig. 6(b).

First, the estimated maximum height of the water displacement is approximately $20 \mu \mathrm{m}$, with the joint angle inversion. The same estimation with the amplitude inversion is around $40 \mu \mathrm{m}$, which is of the same order of magnitude.

Second, a more thorough comparison shows that the use of the angle variation of the eigenbeams yields an inversion of better quality than the amplitude variation. A measurement of the inversion residue variance is performed on the inversion results based on the different eigenbeam observables. To discard the surface wave and measure only the inversion residue, the values inside the red rectangle in Fig. 6 are taken. The inversion residue variance of each inversion is given in Table I, including travel-time inversions, for the sake of completeness. 
TABLE I. Table of the inversion residue variance for the inversions of the eigenbeam amplitude, travel-time, angles taken separately, and joint angles. The inversion residue variance is calculated in the red rectangle shown in Fig. 6.

\begin{tabular}{lcccr}
\hline \hline Eigenbeam observable & Amplitude & Travel-time & Emitting angle & Receiving angle \\
\hline Residue variance & $2.09 \times 10^{-11}$ & $2.45 \times 10^{-11}$ & $6.42 \times 10^{-12}$ & $5.12 \times 10^{-12}$ \\
\hline \hline
\end{tabular}

The results show that the joint angle inversion performs better than the single angle inversion when considering residue variance with a value that halved. This can be expected, as the joint angle inversion is based on twice as much data as the single angle inversion. Interestingly, the single angle inversion (for either emitting or receiving angles) also shows lower residue variance than the inversion based on the amplitude or travel-time variations. This might be surprising at first sight, as the predicted amplitude SK matches the measured amplitude SK from the FM experiment (Fig. 7). A first explanation for the inversion difference between amplitude and angle might be that amplitude measurements are more sensitive to noise than angle measurements.

Another explanation is that the intrinsic properties of the angles SK provide more resolution for the angle inversions than the inversion based on the amplitude SK. Indeed, the amplitude SK is symmetric relative to the reflection point of the eigenbeam [Fig. 7(a)] when the angles SK shows an anti-symmetrical behavior with respect to the reflection point of the eigenbeam (Fig. 3), the SK being null at the reflection point. Moreover, the angles SK show more zero-crossings than the amplitude SK, with therefore faster oscillations and higher spatial resolution. In short, the spatial gradient of the SK leading to faster oscillations of the angles SK here appears to be the key for improved resolution of the inversion result for the surface perturbation.

In conclusion, the use of the emitting and receiving angles in a joint inversion provides a good method for inverting an unknown surface perturbation in the IP experiment. The qualitative and quantitative validation of the inversion comes from independent optical measurements described in a previous study (van Baarsel et al., 2019).
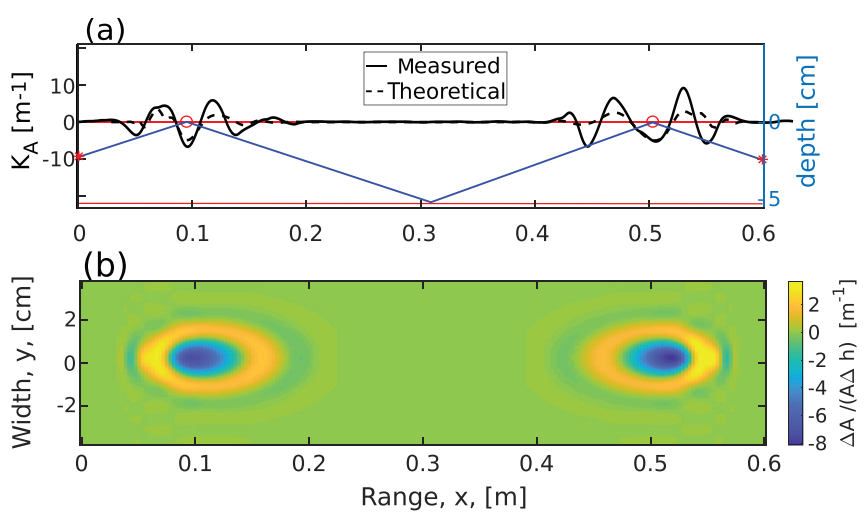

FIG. 7. (Color online) SK for the amplitude of the transmitted signal. (a) Central line of the theoretical SK $(y=0)$, to be compared with Fig. 3. The corresponding eigenray $\left(\left[\theta_{e}, \theta_{r}, t\right]=[-14.8 \mathrm{deg},-14.8 \mathrm{deg}, 427.3 \mu \mathrm{s}]\right)$ is shown under the SK on a different axis. (b) Top-view of the amplitude SK on the whole surface of the waveguide, to be compared with Fig. 4.

\section{CONCLUSION}

This study uses for the first time the variations of the emitting and receiving angles of a set of eigenbeams in an ultrasonic waveguide to accurately invert a dynamic surface perturbation of the waveguide. The 2D SKs for both the emitting and receiving angles link the observable variations to the perturbations of the surface height at the waveguide interface. The first experimental set-up allows us to measure the angles SK using a controlled perturbation of the surface. The experimental results are in good agreement with the theory. The second experiment is used to dynamically invert an unknown perturbation. The results show significant improvement in the joint angles inversion results compared to previous studies based solely on the wavefield amplitude variation.

\section{ACKNOWLEDGMENTS}

This study was supported by Direction Générale de l'Armement (DGA). ISTerre is part of Labex OSUG@2020. This study was performed within the framework of the LABEX Celya (ANR-10-LABX-0060) of Université de Lyon, within the program "Investissement d'Avenir" (ANR16-IDEX-0005) operated by the French National Research Agency (ANR).

\section{APPENDIX A: CALCULATION OF THE COORDINATES IN THE UNWRAPPED SPACE}

The $z$-coordinate of the receiving subarray center $z_{r, \text { image }}$ is defined in the unwrapped space and is calculated from the total number of bottom and surface reflections and the geometry of the waveguide (Fig. 2). Assuming parallel waveguide interfaces, the position of the receiving array in the unwrapped space can be approximated by

$$
\begin{aligned}
& z_{r, \text { image }} \approx-\ell \tan \left(\theta_{e}\right)+z_{e}, \quad \text { if } \theta_{e}>0, \\
& z_{r, \text { image }} \approx \ell \tan \left(\theta_{e}\right)-z_{e}, \quad \text { if } \theta_{e}<0 .
\end{aligned}
$$

The value $r_{z}^{\prime}$ in Eqs. (5a) and (5b) also corresponds to the coordinate along the interface in the unwrapped space for each reflection. Therefore, it is necessary to take into account the number $n$ and the order of the surface reflections for each eigenbeam. For instance, if the emitting angle is positive $\left(\theta_{e}>0\right)$, the first reflection of the eigenbeam will occur at the surface of the waveguide; if it is negative $\left(\theta_{e}<0\right)$, the beam will first reflect at the bottom.

For these reasons, we have the following expression for $r_{z}^{\prime}$, for the $n$th reflection at the surface of the waveguide: 


\section{JASA}

https://doi.org/10.1121/10.0002447

$$
\begin{aligned}
r_{z}^{\prime} & =\left|z_{\text {surf }}-z_{e}\right| \\
& = \begin{cases}\left|2 h(n-1)-z_{e}\right| & \text { if } \theta_{e}<0 \\
\left|2 h n-z_{e}\right| & \text { if } \theta_{e}>0,\end{cases}
\end{aligned}
$$

where $h$ is the height of the waveguide, $z_{e}$ is the position along $z$ of the emitting subarray center, and $z_{\text {surf }}$ is the position along $z$ of the surface in the unwrapped space.

The spatial extent of the SK is to be determined from the bandwidth of the signal and the incident angle of the eigenbeam on the interface. More precisely, considering the Fresnel Zone radius from radar theory $\sqrt{\lambda R}$, where $R$ is the distance between the arrays and $\lambda$ is the central wavelength, the extent of the surface SK for each reflection can be well approximated by

$$
\Delta \mathbf{r}^{\prime} \approx \frac{\sqrt{\lambda R}}{\sin \theta_{e}} \frac{f}{\Delta f},
$$

where $\Delta f / f$ is the normalized bandwidth. For instance, using the numbers for the SK shown in Fig. 3, we have $\Delta \mathbf{r}^{\prime} \approx 0.19 \mathrm{~m}$ centered on each reflection point of the eigenray at the surface.

\section{APPENDIX B: CALCULATION OF THE HESSIAN MATRIX AND ITS INVERSE}

The Hessian matrix is the second-order derivative matrix of the pressure field, i.e.,

$$
\mathbf{H}(p)=\left(\begin{array}{ccc}
\frac{\partial^{2}}{\partial \theta_{e} \theta_{e}} & \frac{\partial^{2}}{\partial \theta_{e} \theta_{r}} & \frac{\partial^{2}}{\partial \theta_{e} t} \\
\frac{\partial^{2}}{\partial \theta_{r} \theta_{e}} & \frac{\partial^{2}}{\partial \theta_{r} \theta_{r}} & \frac{\partial^{2}}{\partial \theta_{r} t} \\
\frac{\partial^{2}}{\partial t \theta_{e}} & \frac{\partial^{2}}{\partial t \theta_{r}} & \frac{\partial^{2}}{\partial t t}
\end{array}\right) p .
$$

To compute the Hessian matrix, we first look for an analytical expression of the unperturbed acoustic pressure field $p_{0}^{D B F}\left(\theta_{e}, \theta_{r}, \omega\right)$. In the unwrapped space, $p_{0}^{D B F}\left(\theta_{e}, \theta_{r}, \omega\right)$ results from the convolution of the wave propagation in the homogeneous medium and the beamforming on the arrays.

The beamforming process can be seen as the interaction of an impinging acoustic wave with an array of sensors in that it is analogous with the phenomenon caused by a diffraction grating in optics (see, e.g., Born and Wolf, 1999, pp. 402-405). Therefore, although Eq. (1) is the most general equation for the DBF wavefield, its expression can be simplified in the case of a quasi-plane wave on two uniform linear arrays as

$$
p_{0}^{D B F}\left(\theta_{e}, \theta_{r}, \omega\right)=p_{0}\left(z_{e, 0}, z_{r, 0}, \omega\right) \cdot B\left(\theta_{e}, \theta_{0}\right) \cdot B\left(\theta_{r}, \theta_{0}\right),
$$

where $p_{0}\left(z_{e, 0}, z_{r, 0}, \omega\right)$ is the acoustic pressure field between the center of the emitting and receiving arrays, and $\theta_{0}$ is the angle between the array and the eigenbeam in the unwrapped space. Note that in this case, $\theta_{0}=\theta_{e}=\theta_{r}$. The function $B\left(\theta, \theta_{0}\right)$ is defined as

$$
B(\theta, \theta)=\frac{\sin \left[\frac{\pi}{\lambda} N d\left(\sin \theta-\sin \theta_{0}\right)\right]}{\sin \left[\frac{\pi}{\lambda} d\left(\sin \theta-\sin \theta_{0}\right)\right]} .
$$

For a pressure field calculated near the eigenbeam emitting and receiving angles, i.e., $\theta \approx \theta_{0}+\Delta \theta$, we can approximate the following:

$$
\sin \theta-\sin \theta_{0} \approx \Delta \theta \cos \theta_{0} .
$$

The characteristic angle in the DBF space is the halfwidth of the eigenbeam spot. In the present experimental configuration, $\Delta \theta \approx 3 \mathrm{deg}$ for each eigenbeam [see Fig. 4(b) from Roux and Nicolas, 2014), which validates the above approximation.

If we rewrite this more compactly as $\theta=\Delta \theta$, we get the expression for the pressure field between two arrays,

$$
\begin{aligned}
p_{0}^{D B F}\left(\theta_{e}, \theta_{r}, \omega\right)= & p_{0}\left(z_{e, 0}, z_{r, 0}, \omega\right) \\
& \times \frac{\sin \left[\frac{\pi}{\lambda} N d \theta_{e} \cos \theta_{0}\right]}{\sin \left[\frac{\pi}{\lambda} d \theta_{e} \cos \theta_{0}\right]} \\
& \times \frac{\sin \left[\frac{\pi}{\lambda} N d \theta_{r} \cos \theta_{0}\right]}{\left.\sin \left[\frac{\pi}{\lambda} d \theta_{r} \cos \theta_{0}\right)\right]} .
\end{aligned}
$$

To compute the Hessian, we need to express the derivatives of the function

$$
f(x)=\frac{\sin (a x)}{\sin (b x)},
$$

where $a=N b$ and $b=(\pi d / \lambda) \theta \cos \theta_{0}$.

The first derivative is

$$
f^{\prime}(x)=\csc (b x)[a \cos (a x)-b \sin (a x) \cot (b x)],
$$

and the second derivative is

$$
\begin{aligned}
f^{\prime \prime}(x)= & \csc (b x)\left[\sin (a x)\left(-a^{2}+b^{2} \cot ^{2}(b x)+b^{2} \csc ^{2}(b x)\right)\right. \\
& -2 a b \cos (a x) \cot (b x)],
\end{aligned}
$$

where the following functions are used:

$$
\begin{aligned}
& \csc (x)=\frac{1}{\sin x}, \\
& \cot (x)=\frac{\cos x}{\sin x} .
\end{aligned}
$$

Interestingly, we note that 


$$
\begin{aligned}
& \lim _{x \rightarrow 0} f^{\prime}(x)=0, \\
& \lim _{x \rightarrow 0} f^{\prime \prime}(x)=\frac{N b^{3}}{3 b}-\frac{N^{3} b^{3}}{3 b} \approx \frac{N^{3} b^{2}}{3},
\end{aligned}
$$

which means that the Hessian becomes a diagonal matrix when the pressure field is computed on the eigenray that joins the two emitting and receiving arrays. This is indeed the case, as we compute the Hessian matrix for the unperturbed eigenbeam. The Hessian can therefore be written as

$$
\mathbf{H}\left(p_{0}^{D B F}\right)=\left[\begin{array}{ccc}
\frac{N^{3} b^{2}}{3} & 0 & 0 \\
0 & \frac{N^{3} b^{2}}{3} & 0 \\
0 & 0 & -\omega^{2}
\end{array}\right] \times p_{0}^{D B F} .
$$

The inverse of the Hessian matrix is then

$$
\mathbf{H}^{-1}\left(p_{0}^{D B F}\right)=\frac{1}{p_{0}^{D B F}}\left[\begin{array}{ccc}
\frac{3}{N^{3} b^{2}} & 0 & 0 \\
0 & \frac{3}{N^{3} b^{2}} & 0 \\
0 & 0 & -\frac{1}{\omega^{2}}
\end{array}\right] .
$$

From Eq. (B13), we immediately see that the three observables $\left[\theta_{e}, \theta_{r}, t\right]$ are uncoupled and can be separated in the forward and inverse problem.

\section{APPENDIX C: CALCULATION OF THE GRADIENT OF THE PRESSURE FIELD VARIATION}

The expression of the gradient $\nabla(\delta p)$ can be obtained by differentiation of Eq. (4). From Eqs. (5a) and (5b), we obtain,

$$
\begin{aligned}
& \frac{\partial}{\partial \theta_{e}} K^{D B F}\left(\theta_{e}, \theta_{r}, \mathbf{r}^{\prime}, \omega\right) \\
& \quad=\imath k\left[-r_{x}^{\prime} \sin \theta_{e}+\left(z_{e}-r_{z}^{\prime}\right) \cos \theta_{e}\right] K^{D B F}\left(\theta_{e}, \theta_{r}, \mathbf{r}^{\prime}, \omega\right),
\end{aligned}
$$

$$
\begin{aligned}
\frac{\partial}{\partial \theta_{r}} & K^{D B F}\left(\theta_{e}, \theta_{r}, \mathbf{r}^{\prime}, \omega\right) \\
= & l k\left[-\left(\ell-r_{x}^{\prime}\right) \sin \theta_{r}+\left(z_{r, \text { image }}-r_{z}^{\prime}\right) \cos \theta_{r}\right] \\
& \times K^{D B F}\left(\theta_{e}, \theta_{r}, \mathbf{r}^{\prime}, \omega\right) .
\end{aligned}
$$

The gradient of the pressure variation can therefore be written as

$$
\begin{aligned}
\nabla(\delta p) & =\left[\begin{array}{c}
\partial / \partial \theta_{e} \\
\partial / \partial \theta_{r} \\
\partial / \partial t
\end{array}\right] \delta p \\
& =\left[\begin{array}{c}
\imath k\left(-\sin \left(\theta_{e}\right) r_{x}^{\prime}+\cos \left(\theta_{e}\right)\left(z_{e}-r_{z}^{\prime}\right)\right) \\
\imath k\left(-\sin \left(\theta_{r}\right)\left(\ell-r_{x}^{\prime}\right)+\cos \left(\theta_{r}\right)\left(z_{r, \text { image }}-r_{z}^{\prime}\right)\right) \\
-\imath \omega
\end{array}\right] \delta p .
\end{aligned}
$$

Aulanier, F., Nicolas, B., Roux, P., and Mars, J. I. (2013). "Time-angle sensitivity kernels for sound-speed perturbations in a shallow ocean," J. Acoust. Soc. Am. 134(1), 88-96.

Beydoun, W. B., and Tarantola, A. (1988). "First Born and Rytov approximations: Modeling and inversion conditions in a canonical example," J. Acoust. Soc. Am. 83(3), 1045-1055.

Born, M., and Wolf, E. (1999). Principles of Optics (Cambridge University Press, Cambridge, UK), pp. 402-405.

Cornuelle, B. D. (1982). "Acoustic tomography," IEEE Trans. Geosci. Remote Sens. 20(3), 326-332.

Iturbe, I., Roux, P., Virieux, J., and Nicolas, B. (2009). "Travel-time sensitivity kernels versus diffraction patterns obtained through double beamforming in shallow water," J. Acoust. Soc. Am. 126(2), 713-720.

Krüger, F., Weber, M., Scherbaum, F., and Schlittenhardt, J. (1993). "Double beam analysis of anomalies in the core-mantle boundary region," Geophys. Res. Lett. 20(14), 1475-1478, https://doi.org/10.1029/93GL01311.

Marandet, C., Roux, P., Nicolas, B., and Mars, J. (2011). "Target detection and localization in shallow water: An experimental demonstration of the acoustic barrier problem at the laboratory scale," J. Acoust. Soc. Am. 129(1), 85-97.

Mayer, W. G. (1963). "Reflection and refraction of mechanical waves at solid-liquid boundaries. II,” J. Appl. Phys. 34(11), 3286-3288.

Munk, W., and Wunsch, C. (1979). "Ocean acoustic tomography: A scheme for large scale monitoring.," Deep Sea Res. Part A. Oceanogr. Res. Papers 26(2), 123-161.

Pekeris, C. L. (1948). "Theory of propagation of oxplosive sound in shallow water," in Geological Society of America Memoirs (Geological Society of America, Washington, DC), p. 116.

Roux, P., Cornuelle, B. D., Kuperman, W. A., and Hodgkiss, W. S. (2008). "The structure of raylike arrivals in a shallow-water waveguide," J. Acoust. Soc. Am. 124(6), 3430-3439.

Roux, P., and Fink, M. (2000). "Time reversal in a waveguide: Study of the temporal and spatial focusing," J. Acoust. Soc. Am. 107(5), 2418-2429.

Roux, P., and Nicolas, B. (2014). "Inverting for a deterministic surface gravity wave using the sensitivity-kernel approach," J. Acoust. Soc. Am. 135(4), 1789-1799.

Sarkar, J., Marandet, C., Roux, P., Walker, S., Cornuelle, B. D., and Kuperman, W. A. (2012). "Sensitivity kernel for surface scattering in a waveguide," J. Acoust. Soc. Am. 131(1), 111-118.

Skarsoulis, E. K., and Cornuelle, B. D. (2004). "Travel-time sensitivity kernels in ocean acoustic tomography," J. Acoust. Soc. Am. 116(1), 227-238.

Snieder, R., and Nolet, G. (1987). "Linearized scattering of surface waves on a spherical earth,” J. Geophys. 61(1), 55-63.

van Baarsel, T., Roux, P., Mars, J. I., Bonnel, J., Arrigoni, M., Kerampran, S., and Nicolas, B. (2019). "Dynamic imaging of a capillary-gravity wave in shallow water using amplitude variations of eigenbeams," J. Acoust. Soc. Am. 146(5), 3353-3361. 